\title{
Region-based perceptual grouping: a cooperative approach based on Dempster-Shafer theory
}

\author{
Nicolas Zlatoff ${ }^{a}$, Bruno Tellez ${ }^{a}$ and Atilla Baskurt ${ }^{a}$ \\ ${ }^{a}$ LIRIS, UMR 5203 Université Lyon 1, \\ 43 boulevard du 11 novembre 1918, 69622 Villeurbanne Cedex, France
}

\begin{abstract}
As segmentation step does not allow to recover semantic objects, perceptual grouping is often used to overcome segmentation's lacks. This refers to the ability of human visual system to impose structure and regularity over signal-based data. Gestalt psychologists have exhibited some properties which seem to be at work for perceptual grouping and some implementations have been proposed by computer vision. However, few of these works model the use of several properties in order to trigger a grouping, even if it can lead to an increase in robustness. We propose a cooperative approach for perceptual grouping by combining the influence of several Gestalt properties for each hypothesis. We make use of Dempster-Shafer formalism, as it can prevent conflicting hypotheses from jamming the grouping process.
\end{abstract}

Keywords: Perceptual Grouping, Segmentation, Dempster-Shafer, Gestalt

\section{INTRODUCTION}

Computer vision aims at extracting a symbolic description from signal-based raw data. It proceeds by a hierarchy of treatments, handling more and more abstract tokens. One important step is perceptual grouping, which refers to the ability of human visual system to impose structure and regularities over several stimuli. Hence, perceptual grouping aims at extracting salient structures from images, which could be further handled by interpretative tasks in order to form semantic objects.

Basically, two kinds of treatments could be seen, though strongly linked. On the one hand, top-down (attentive) processes need external knowledge in order to perform goal-oriented tasks. Such knowledge could be of various kinds (eg declarative in ontological formats or integrated in processes). On the other hand, bottom-up (pre-attentive) processes handle only signal-based data, without any additional knowledge. We focus in this article on this kind of treatments.

Among several approaches of perceptual grouping, the simplest step consists in grouping pixels into various structures based on low-level descriptors such as color or texture ${ }^{1}{ }^{2}$ However, those approaches are quite limited as they cannot extract structures composed of several parts with very different low-level descriptors for each of them. As a lot of real objects are just of that kind, it is a strong limitation. That is why some other works have tried to apply other criteria for subsequent grouping. For instance, they try to group two regions with close and continuous borders, as they may be two parts of an object. Other criteria have been used such as compactness, similarity, symmetry. Early in the 20th century, psychologists from Gestalt school ${ }^{3}$ have already formalized the use of such criteria for grouping. They have argued that vision proceeds by successive groupings, involving some basic properties: proximity, similarity, closure (compactness), continuity and symmetry for instance. Then, several works issued from computer vision have proposed implementations of those criteria. They often rely on contour-based segmented image. Besides, they make use for each grouping, either of one property or of several ones but in a static, pre-defined configuration. We introduce a new framework for bottom-up (pre-attentive) perceptual organization, which emphasizes the cooperation of several criteria for each grouping hypothesis. We use Dempster-Shafer theory of evidence to model this interaction. As our aim is to improve the quality of a segmentation step, we rely on a previously segmented image.

The scope of this article will be as follows: next section will review related works on perceptual grouping. Section 2 presents the model for perceptual grouping, and basics of Dempster-Shafer formalism. After detailing Gestalt measurements in section 3, we present several results in section 4.

Further author information: (Send correspondence to Nicolas Zlatoff.)

Nicolas Zlatoff: E-mail: nicolas.zlatoff@liris.cnrs.fr, Telephone: + 33 (0)4 72448395 


\subsection{Related works}

Sarkar and Boyer ${ }^{4}$ present a survey on perceptual grouping. They argue that a lot of approaches make use of Gestalt principles such as proximity, similarity, closure, continuity and symmetry. Let first note that most of these approaches focus on contour-based primitives, which seems to us a limitation. As a matter of fact, regionbased structures may be more adapted for some kind of properties such as similarity or closure (compactness). That is why we rely on a region-based segmented image for perceptual grouping. This allows us to use both region and contour clues whenever they are the most appropriate.

Lowe $^{5}$ may be the first one who made use of some of Gestalt properties in order to perform perceptual groupings. His main contribution is the formalization of non-accidentalness principle, so as to compute the significance of a grouping: it is inversely proportional to its prior probability of occurrence. For instance, as three points are very unlikely to be aligned in an image, if such an alignment occurs, it may reflect a salient structure in the image. Moisan and Desolneux ${ }^{6}$ formalized an analogous strategy to infer groupings' saliency based on Helmholtz principle. We make also use of the principle of non-accidentalness in our framework. However, as previously mentioned by Moisan and Desolneux, ${ }^{6}$ several properties are often at work for a grouping to occur. Very few approaches take this information into account, although it can allow a strong increase in robustness. As a matter of fact, it can prevent one single property from triggering a grouping. We model such cooperation in our framework.

Sarkar and Boyer ${ }^{78}$ propose a hierarchical framework of grouping, based on contour segmentation. Here, tokens of increasingly complexity are iteratively grouped, thanks to a static hierarchy of treatments. Inferences on geometric properties are further handled ${ }^{7}$ thanks to bayesian nets. Similar approaches can be found ${ }^{91011}$ with different global control imposed on groupings such as Markov Random Fields (MRF) ${ }^{9}$ or fuzzy logics. ${ }^{10}$ However, those works always extract static, pre-defined structures, which is a strong limitation.

In order to handle more complex interactions, Murino et al. ${ }^{12}$ model in a graph a set of contours linked together by grouping hypothesis. A MRF is then used in order to find the most stable state. However, one Gestalt property is used as a potential activator for each grouping hypothesis. Idrissi et al. ${ }^{13}$ use a heuristic in order to reduce an analogous graph, with properties of proximity, similarity and closure at work for each hypothesis. However, they use a weighted sum to combine properties' effects and thus do not handle precisely the interaction. The same holds for works from Luo and Guo, ${ }^{14}$ who uses a MRF with a greedy algorithm for grouping.

In fact, classical bayesian theories handle with difficulties interactions of several properties on one given hypothesis. As a matter of fact, properties are likely to contradict each other and it is therefore difficult to combine their influence. On the contrary, Dempster-Shafer theory ${ }^{15}$ is especially well-suited for such needs, as it allows to handle belief rather than probabilities. The main difference between those two concepts relies in the fact that a portion of belief could be committed into one hypothesis without committing the remainder into the hypothesis' negation. Hence, several criteria may not contradict each other. Vasseur et al. ${ }^{16}$ make use of Dempster-Shafer formalism for perceptual grouping on contour primitives. However, their model is just a bayesian view of Dempster-Shafer theory and still leads to conflicting sets of hypotheses. On the contrary, our framework does model cooperation between Gestalt properties and prevents conflict between them from jamming the groupings.

\subsection{Framework for perceptual grouping}

We propose a cooperative framework for perceptual grouping. We rely on a region-based segmented image, and we impose several Gestalt properties to be at work in order to trigger a grouping. This model leads to a strong increase of robustness as it prevents one single property from triggering a grouping.

Grouping hypotheses are generated from a Region Adjacency Graph (RAG) where a vertex stands for a region while an edge is instantiated between two regions when those are adjacent. Hence, edges from RAG represent grouping hypotheses. Then, each Gestalt property leads to a partial belief for grouping on each hypothesis. Those beliefs are then combined together in order to assign each hypothesis with a global belief for grouping. Finally, RAG is reduced, based on those global beliefs. 


\section{MODEL FOR PERCEPTUAL GROUPING}

Dempster-Shafer theory is a probabilistic theory of evidence which allows a flexible modelling of uncertainty. Thus, it is particularly well-designed when working with incomplete data. Here, the incomplete data are the extent to which a Gestalt property does not favour a grouping. As a matter of fact, we only model the extent to which a property does favour a grouping. As we will see below, this model prevents conflicting hypotheses from jamming the whole process.

Basically, when classical bayesian theories commit a probability $x$ into one hypothesis $A$, they also commit the remainder $1-x$ into its negation, that is $\bar{A}$. On the contrary, Dempster-Shafer framework allows one to commit a portion $x$ into $A$ without committing the remainder $1-x$ into $\bar{A}$.

Besides, Dempster-Shafer theory allows one to compute a combined belief on a set of hypotheses, from several other partial points of view. This is especially well-suited in our case, as each Gestalt property gives a partial belief for each grouping hypothesis. What we need is then a formalism to compute a combined belief for groupings, taken into account all Gestalt properties. Further details on Dempster-Shafer formalism are presented in the next paragraphs.

\subsection{Basics of Dempster-Shafer theory}

Let $\Theta$ be a finite set of mutually exclusive hypotheses $\left\{H_{1}, H_{2}, \ldots, H_{n}\right\}$, called frame of discernment. The set of all subsets of $\Theta$ is denoted $2^{\Theta}$.

We call basic probability assignment (bpa) a function $m: 2^{\Theta} \rightarrow\left[\begin{array}{ll}0 & 1\end{array}\right]$ which satisfies the following conditions:

$$
m(\oslash)=0 \quad \sum_{A \subset \Theta} m(A)=1
$$

For each subset $A$ of $\Theta, m(A)$ could be interpreted as one's degree of belief that the truth lies exactly in $A$. Basically, this does not correspond to one's total belief that the truth lies in $A$, since we must add all exact belief on hypotheses $B$ that imply $A$. Hence, we define $\operatorname{Bel}(A)$, total belief in $A$ :

$$
\operatorname{Bel}(A)=\sum_{B \subset A} m(B)
$$

This function $B e l: 2^{\Theta} \rightarrow\left[\begin{array}{ll}0 & 1\end{array}\right]$ is called belief function. Note that, in general, one does not have: $\operatorname{Bel}(A)=$ $m(A)$.

When building a belief function (or, in practice, its associated bpa), one must commit belief over the set of hypotheses $H_{i}$ from $\Theta$ or over a subset $A$ of $\Theta$ when uncertainty prevents us from being more precise. Hence, a subset $A$ of $\Theta$ is called focal element if and only if a portion of belief has been committed over it, that is: $m(A)>0$.

\subsection{Combining belief functions}

Dempster's rule of combination allows one to combine several belief functions over the same set of hypotheses, in order to infer a belief function that takes into account all the influences of the others.

Let $m_{1}$ and $m_{2}$ be two bpa-s associated with belief functions $B e l_{1}$ and $B e l_{2}$ respectively, over the same frame of discernment $\Theta$. Dempster's rule of combination enables us to compute the orthogonal sum of $B e l_{1}$ and $B e l_{2}$, denoted $B e l_{1} \oplus B e l_{2}$, which represents the combined belief committed over $\Theta$, given the influence of both $B e l_{1}$ and $B e l_{2}$. Let us denote $A_{i}$ the focal elements of $B e l_{1}$ while $B_{j}$ those of $B e l_{2}$. If $\sum_{A_{i} \cap B_{j}=\varnothing} m_{1}\left(A_{i}\right) m_{2}\left(B_{j}\right)<1$, then the function $m: 2^{\Theta} \rightarrow\left[\begin{array}{ll}0 & 1\end{array}\right]$ defined by

$$
m(\oslash)=0 \quad m(C)=\frac{\sum_{A_{i} \cap B_{j}=C} m_{1}\left(A_{i}\right) m_{2}\left(B_{j}\right)}{1-\sum_{A_{i} \cap B_{j}=\oslash} m_{1}\left(A_{i}\right) m_{2}\left(B_{j}\right)}
$$




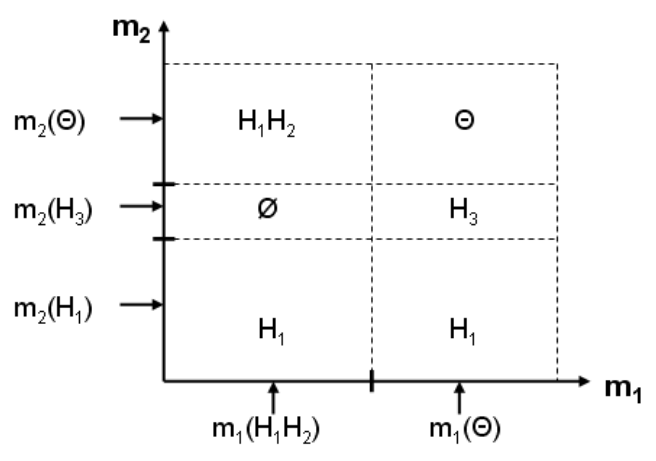

(a)

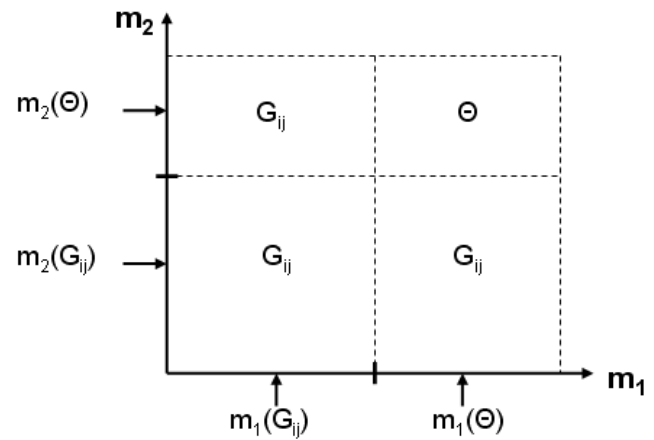

(b)

Figure 1. Example of Dempster's rule of combination for two belief functions (a) and in our model (b). Note that there is no conflict among our hypotheses in (b).

for all non-empty $C \subset \Theta$, is a bpa. Its associated belief function is called orthogonal sum of $B e l_{1}$ and $B e l_{2}$.

The situation could be graphically illustrated in figure $1(\mathrm{a})$. Let us suppose that $\Theta=\left\{H_{1}, H_{2}, H_{3}\right\}$. The first bpa $m_{1}$ commits belief over $A_{1}=H_{1} H_{2}$ and $A_{2}=\Theta$, while second bpa $m_{2}$ commits belief over $B_{1}=H_{1}$, $B_{2}=H_{3}$ and $B_{3}=\Theta$. The resulting bpa $m$ is defined over 6 sets, corresponding to the combination of each focal element. For instance, one has:

$$
m\left(H_{1}\right)=\frac{m_{1}\left(H_{1} H_{2}\right) m_{2}\left(H_{1}\right)+m_{1}(\Theta) m_{2}\left(H_{1}\right)}{1-m_{1}\left(H_{1} H_{2}\right) m_{2}\left(H_{3}\right)}
$$

The positive number $k=\sum_{A_{i} \cap B_{j}=\varnothing} m_{1}\left(A_{i}\right) m_{2}\left(B_{j}\right)$ is called conflict measure and tends towards 1 when belief functions are defined over sets of hypotheses that contradict each other. It corresponds to the case when intersection of hypotheses boils down to $\oslash$. In this case, results from equation (3) are unlikely to be representative, as the conflict may represent a large portion of belief.

\subsection{Handling conflicting hypotheses}

For our purpose, each belief function corresponds to the influence of one Gestalt property. For each edge of the RAG, we consider $\Theta=\left\{G_{i j}, \overline{G_{i j}}\right\}$ where $G_{i j}$ stands for grouping region $i$ and region $j$. Each belief function commits belief over two hypotheses: $G_{i j}$ and uncertainty $\Theta$. The model is illustrated in figure 1(b).

Note that, due to the choice of our focal elements, there is no conflict among our hypotheses $(k=0)$. Hence, results from combination are always representative. This is very different from classical bayesian theories where belief would have been commited both to $G_{i j}$ and to $\overline{G_{i j}}$. In this case, conflicting hypotheses may have appeared, which would result in a possible breakdown for the grouping.

Note also that:

$$
m\left(G_{i j}\right)=m_{1}\left(G_{i j}\right)+m_{2}\left(G_{i j}\right)\left(1-m_{1}\left(G_{i j}\right)\right)
$$

Therefore:

$$
m\left(G_{i j}\right)>m_{1}\left(G_{i j}\right) \text { and } m\left(G_{i j}\right)>m_{2}\left(G_{i j}\right)
$$

That means that beliefs from several belief functions over one grouping hypothesis tend to reinforce themselves. Hence, when different Gestalt properties are activated from one grouping hypothesis they act in favour 


\section{$\bullet \bullet \bullet \bullet \bullet$ \\ (a)

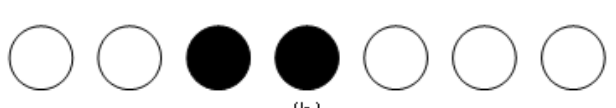

(b)

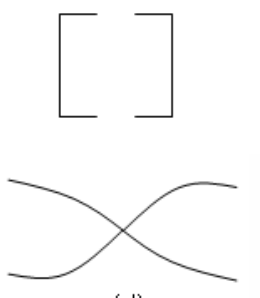

(d)

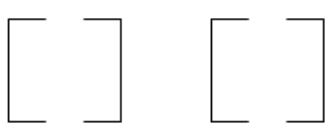

(c)

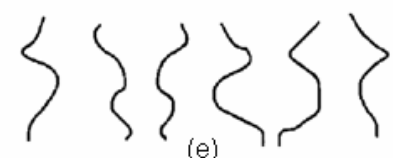

Figure 2. Examples of Gestalt properties of grouping (adapted from D. Lowe ${ }^{5}$ ): proximity (a), similarity (b), closure (c), continuity (d) and symmetry (e).

of the grouping in a cooperative way. Numerical examples of several combinations are shown in figure 4 from section 'Results'.

As our purpose is to combine more than two belief functions, note that those results can be generalized for a set of $n$ belief functions by iteratively handle equation (3).

\section{GESTALT MEASUREMENTS}

This section will explain how the several grouping hypotheses are characterized, considering each Gestalt property. There is no real consensus, even inside Gestalt school, ${ }^{3}$ on how many properties are involved during perceptual grouping, and on how they should be implemented. Examples of properties are shown in figure 2. For instance, one tends to group circles from (b) according to a color-based similarity. In the same way, lines from (c) tend to be grouped in order to form close shapes, even if they are equally-spaced. Note also the continuity property which states that patterns from (d) are viewed as two continuous lines intersecting rather than two adjacent cups.

We propose to characterize a grouping hypothesis with three different properties, which seem to be quite relevant: proximity, similarity, closure (compactness) and continuity/symmetry. Note that property of proximity is directly handled by the use of a RAG during hypothesis extraction.

\subsection{Similarity}

Similarity is thought from the point of view of the descriptors used during segmentation step. As a matter of fact, each segmented region $R_{i}$, handles a set of descriptors $d_{i, k}$ that take homogeneous values among each of its pixels. Hence, we define the similarity measure as a classical distance in Euclidean space:

$$
M_{1}\left(G_{i j}\right)=\left(\sum_{k}\left(d_{i, k}-d_{j, k}\right)^{2}\right)^{1 / 2}
$$

When the descriptors used are color-based, we rely on the CIE Lab color space. In this way, the Euclidean distance reflects explicitly the perceptual distance between the two sets of colors. $M_{1}\left(G_{i j}\right)$ tends towards 0 when the sets of descriptors for the two regions tend to be identical.

\subsection{Closure-Compactness}

According to, ${ }^{3}$ closure property tends to favour the perception of simple, closed and regular object. It has often been reduced in contour-based approaches only to closed objects, but it also refers to compactness. That is why we introduce:

$$
M_{2}\left(G_{i j}\right)=\left|1-\frac{\operatorname{area}\left(R_{i}+R_{j}\right)}{\operatorname{area}\left(\operatorname{ellipse}\left(R_{i}+R_{j}\right)\right)}\right|
$$

where $R_{i}+R_{j}$ represents the region issued from the merging of regions $R_{i}$ and $R_{j}$. ellipse $\left(R_{i}+R_{j}\right)$ stands for the ellipse which has the same second order moments as $R_{i}+R_{j}$. We make use of an ellipse as it allows us to approximate a convex hull very quickly considering the computing time. 


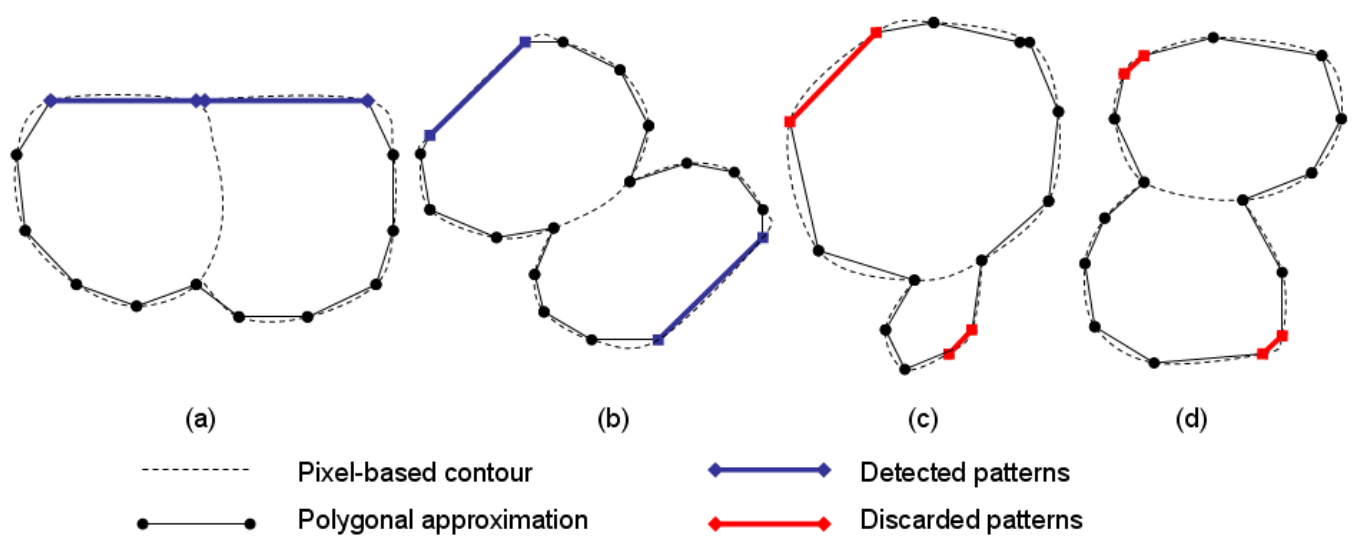

Figure 3. Examples of detected $(\mathrm{a}, \mathrm{b})$ or discarded $(\mathrm{c}, \mathrm{d})$ patterns by continuity/symmetry property. (a) corresponds to continuity among contours while (b) corresponds to parallelism. In (c), involved segments doe not have a similar size in order to trigger a symmetry, while in (d) they are not representative from their regions.

$M_{2}\left(G_{i j}\right)$ tends towards 0 when $R_{i}+R_{j}$ tends to shape as an ellipse with the same second order moments. It therefore favours both groupings which shape like ellipses, and that involve one region strongly bounded by another one.

\subsection{Continuity-Symmetry}

On the contrary to previous properties, continuity and symmetry far more rely on contours of regions than on regions only. We unify them in one single property, considering they both rely on the same kind of notion that is the orientation difference between primitive segments. The main difference is that continuity needs two segments to be close, while symmetry can handle more distant ones. We use a polygonal approximation of regions contours, based on a recursive approximation. ${ }^{17}$ Then, orientation $\theta_{s_{m}}$ is extracted for each segment $s_{m}$ and a global measure is set:

$$
M_{3}\left(G_{i j}\right)=\min _{\left(s_{m}, s_{n}\right) \in\left(\mathcal{S}_{i} \times \mathcal{S}_{j}\right)}\left(\left|\theta_{s_{m}}-\theta_{s_{n}}\right| \alpha_{s_{m}} \alpha_{s_{n}} \beta_{s_{m} s_{n}}\right)
$$

where $\mathcal{S}_{i}$ stands for the set of segments issued from polygonal approximation of $R_{i}$.

$\alpha_{s_{m}}$ and is $\beta_{s_{m} s_{n}}$ are two corrective parameters $\left(\alpha_{s_{m}}>1, \beta_{s_{m} s_{n}}>1\right)$, which prevent the detection of pattern like in figure $3(\mathrm{~d}))$ and $3(\mathrm{c})$ ), respectively.

$$
\alpha_{s_{m}}=\frac{\max _{s_{k} \in \mathcal{S}_{i}}\left(l_{s_{k}}\right)}{l_{s_{m}}} \quad \beta_{s_{m} s_{n}}=\frac{\max \left(l_{s_{m}}, l_{s_{n}}\right)}{\min \left(l_{s_{m}}, l_{s_{n}}\right)}
$$

with $l_{s_{m}}$ the length of segment $s_{m}$. Note that $\alpha_{s_{n}}$ is the analogous term of $\alpha_{s_{m}}$ for region $R_{j}$.

Examples of detected patterns for this continuity/symmetry property are shown in figure 3(a)(b).

\subsection{Normalization of measurements}

In order to normalize raw measurements $M_{k}\left(G_{i j}\right)$ into bpa $m_{k}$, we use the principle of non-accidentalness. ${ }^{5}$ Roughly speaking, it formulates that the significance of a grouping is proportional to the inverse of its prior probability of appearance. For instance, since three points are unlikely to be aligned in an image, if such an alignment is found, it represents a grouping of high significance. Following that idea, we argue that mean value $\overline{M_{k}}$ of a measure $M_{k}\left(G_{i j}\right)$ cannot be regarded as salient. Considering also that raw measurements $M_{k}\left(G_{i j}\right)$ tends to 0 when corresponding Gestalt property tends to be active, we set:

$$
m_{k}\left(G_{i j}\right)=\frac{2}{N}\left[1-\frac{M_{k}\left(G_{i j}\right)}{\overline{M_{k}}}\right] \text { if } M_{k}\left(G_{i j}\right)<\overline{M_{k}} \text { or } m_{k}\left(G_{i j}\right)=0 \text { else }
$$




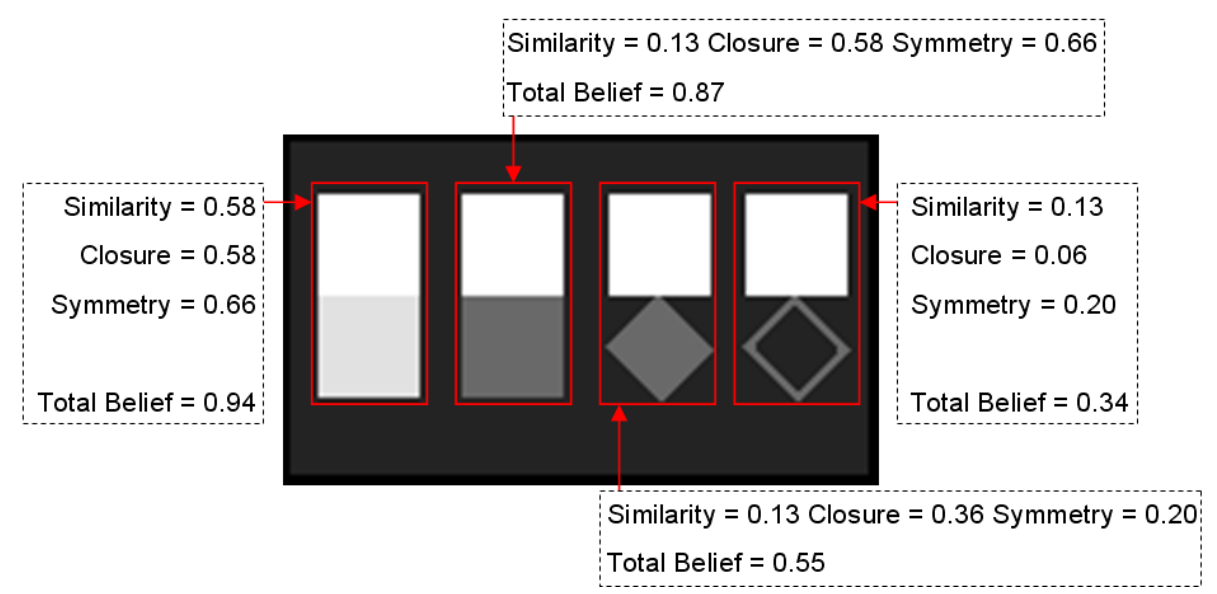

Figure 4. Examples of perceptual grouping with details.

$N$ is the number of Gestalt properties used. The normalization factor $\frac{2}{N}$ ensures that one single property cannot give a combined belief of 1 (total belief) in one hypothesis but rather needs other properties to cooperate with it in order to make a grouping occur.

\section{RESULTS}

Figure 4 shows how our system behaves on artificial images. For each of the four grouping hypotheses (surrounded in red), three partial beliefs are exhibited, correponding to the Gestalt properties of similarity, closure and symmetry. Finally, a total belief for each is computed, based on Dempster's rule. Recall that value of one for belief corresponds to total truth. First of all, we can see that our implementations of each Gestalt properties seems quite correct: symmetries between squares are detected, and so as to the color-based similarity. Note also that closure belief tend to favour compact groupings.

Secondly, we can see that properties tend to reinforce their beliefs. Hence, pattern on the left has the strongest total belief, since all Gestalt properties have been triggered. On the contrary, when some properties disagree on the grouping, our system is able to find a total belief, though, taking into account the conflicting viewpoints. (two patterns on center).

Given a combined belief value for each hypothesis (ie edge), RAG is then iteratively reduced. At that moment, we use a High Confidence First (HCF) algorithm, which iteratively merges regions linked by the edge with the strongest belief associated. This ensures the reduction process to converge, but does not prevent it from falling into local extrema. Further works will be directed in this way. RAG is iteratively reduced until there is no more edge whose combined belief is more than a value (denoted minBelief). This one is linked to the granularity of the grouping. The more it will be, the less the regions will be grouped. Note that the setting of this value is hence quite intuitive for a user.

We have tested our perceptual grouping on previously segmented images. We used a subset of Corel(c) database, segmented by a mean-shift color-based algorithm described by Comaniciu and Meer. ${ }^{18}$ Examples of results are shown in figure 5 where three images are displayed: original image in (a), segmented image in (b) and perceptually grouped image in (c). Results show that perceptual grouping is able to significantly reduce noise issued from segmentation step. Besides, perceptual grouping tends to make semantic objects emerge (fishes in top images, and different vegetables on last two images). Note for example that artefacts due to illumination on last two images are removed thanks to closure property. Note also that artefacts on left of yellow bell pepper (fourth image), issued from segmentation is removed during perceptual grouping.

Computation time depends on the initial segmentation and more precisely on the number of regions. On the subset of images used (500 images), it is less than 1 second on a $3 \mathrm{GHz}$ Pentium 4 for both segmentation and perceptual grouping of one image. Images' size is $256^{*} 384$ pixels. Segmentation step typically produces between 

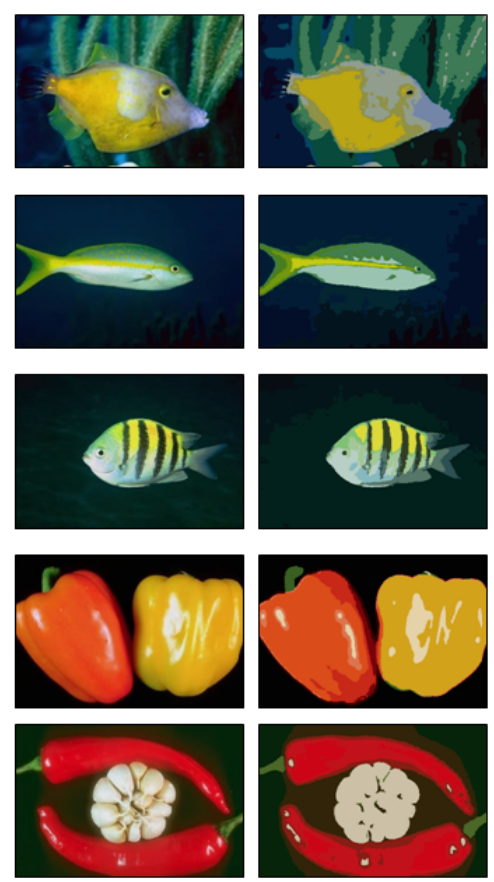

(a)

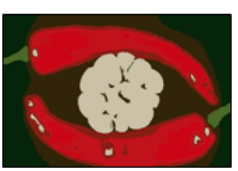

(b)
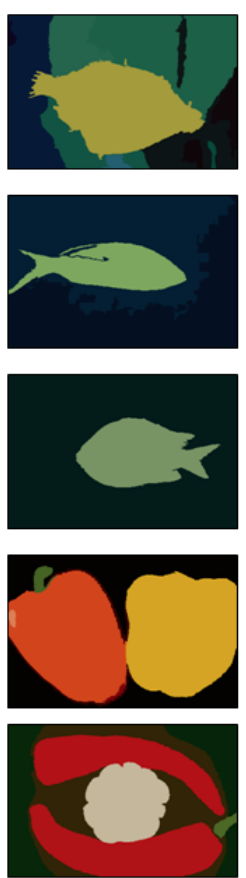

(c)
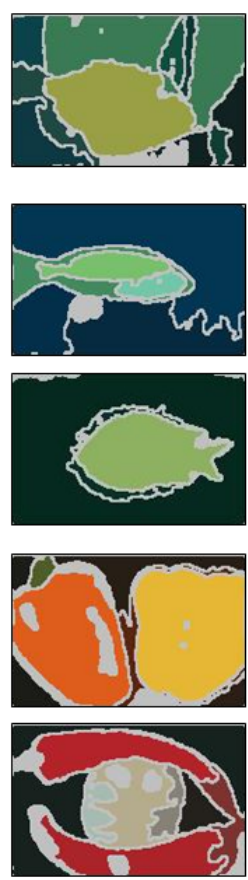

(d)

Figure 5. Examples of results of our perceptual grouping (c) from segmented images (b). Original images are shown on (a). Blobworld segmentation is shown on (d). Note that grey regions in Blobworld corresponds to discarded pixels. Parameter minBelief is set respectively to $50 \%, 40 \%, 35 \%, 62 \%$ and $55 \%$.

200 and 900 regions depending on its content, and perceptual grouping is able to produce a description that handles between 4 and 50 regions.

In order to show the relevancy of perceptual grouping over other methods based on segmentation only (that is: similarity and proximity properties), we have also made comparisons with a well-known segmentation framework which has proven to be efficient: Blobworld system. ${ }^{1}$ It models image with a mixture of Gaussians in a multidimensional space, involving color, texture and position for each pixel and uses expectation-maximization principle in order to estimate the model's parameters. Examples of results are shown in figure 5(d). Note that grey regions in Blobworld results correspond to discarded ones. We can see that our system performs better, in so far that it is able to group regions that have different color or texture descriptors but present a kind of unity though. Hence, the fishes are almost recovered in the first two images because perceptual grouping does not rely solely on low-level descriptors like color or texture. On the contrary, Blobworld's description of first image keep regions near fish's eye and tail separated both from the background and the fish. Besides, the use of continuity or closure property can also help to remove artefacts due to reflections for instance in the last two images.

Other results of perceptual grouping are displayed in figure 6. As content is far more complex, perceptual grouping is not able to fully recover semantic objects. However, groupings make salient structures emerge which may be useful for subsequent treatments of object recognition for instance. First of all, perceptual grouping leads to an extraction of subject from background. In addition, several regions of interest are correctly extracted such as skin, clothes for images of women and wheels or body for cars. Note that girl's hair in third image is wrongly merged during segmentation with a long thin region from background. It is therefore merged with the whole background during perceptual grouping thanks to a combined similarity-closure property. Note also in the second image of cars, the use of both similarity and symmetry for the grouping of the ground, so as to the grouping of the body in the first one. 

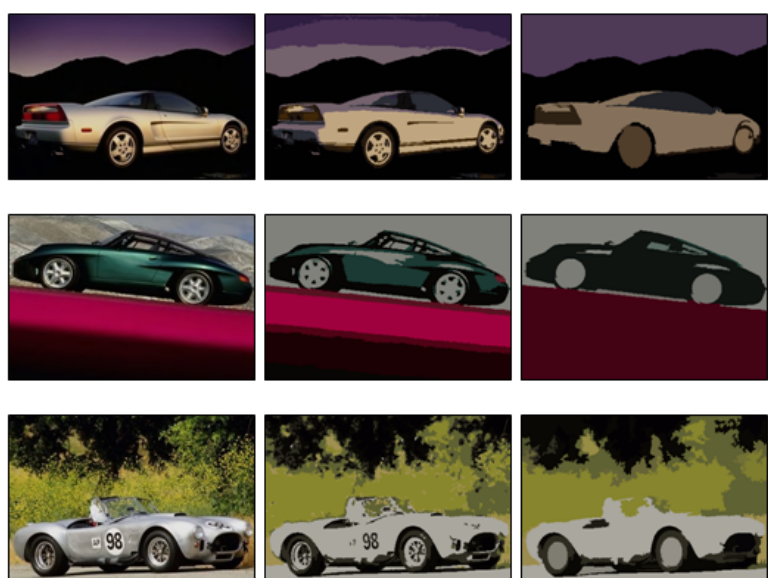

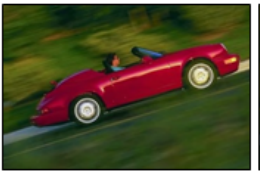

(a)

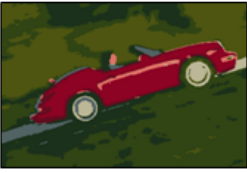

(b)

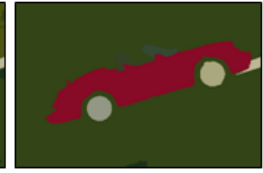

(c)
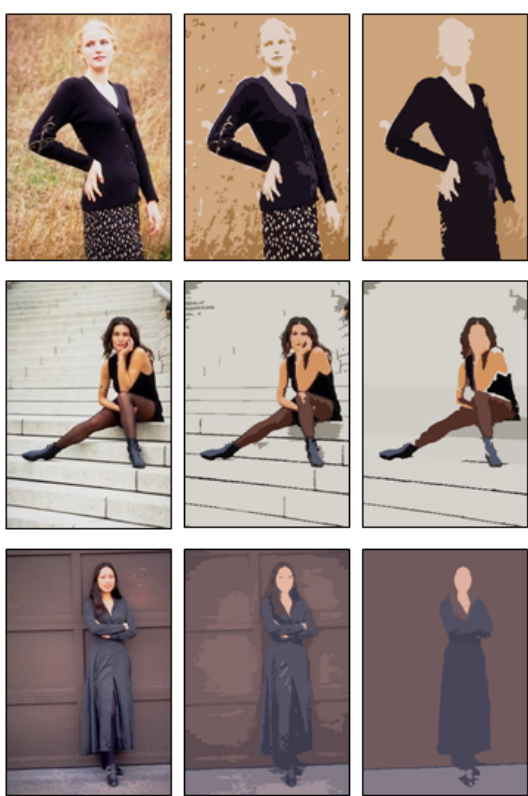

(a)

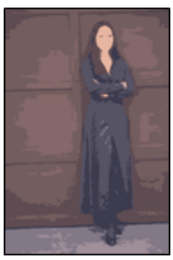

(b)

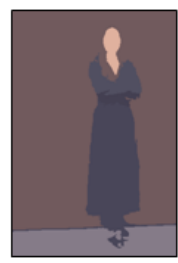

(c)

Figure 6. Examples of results of our perceptual grouping (c) from segmented images (b). Original images are shown on (a). Parameter minBelief is set respectively to $43 \%, 68 \%, 70 \%$ and $62 \%$ for images on the left and to $55 \%, 50 \%$ and $71 \%$ for images on the right.

\section{SUMMARY AND CONCLUSION}

We have presented a new framework for perceptual grouping, on region-based segmented images. One of the originality of our approach is to trigger a grouping when evidence of it appears from multiple sources, that is when several Gestalt-based properties support the grouping. It represents a strong increase in robustness as it prevents one single Gestalt property from triggering a grouping alone. Besides, Dempster-Shafer formalism enables us to model a cooperative framework for Gestalt properties without any conflict between them.

We have also introduced an implementation of several Gestalt laws which seem relevant: proximity, similarity, closure (compactness) and continuity/symmetry.

Results displayed are quite promising and show the increase of quality over segmentation approaches, as our framework allows to merge regions that have very different low-level descriptors but that belong to a common object. Besides, it permits to extract salient structure from background and to handle some artefacts issued from segmentation step.

In further works, we will try to implement one more Gestalt meta-property, which is called Prägnanz and refers to the creation of objects of minimum entropy. We plan for a curvature-based descriptor for doing this. Besides, a special kind of interest will be directed in the reduction process, which can sometimes fall into local extrema. Other graph-based techniques, such as the min-cut algorithm may be useful in order to iteratively partition the graph into subsets so as to minimize the cost from a Gestalt point of view.

We shall also recall that segmentation is not a goal on its own. That is we are currently using perceptual grouping with methods of object recognition. Here, the use of shape descriptors is useful in order to stop or to call into question the perceptual grouping. Promising results have already been obtained in this area.

\section{REFERENCES}

1. C. Carson, S. Belongie, H. Greenspan, and J. Malik, "Blobworld: image segmentation using expectationmaximization and its application to image querying," IEEE Transactions on Pattern Analysis and Machine Intelligence 24(8), pp. 1026-1038, 2002. 
2. J. Wang, J. Li, and G. Wiederhold, "Simplicity: Semantics-sensitive integrated matching for picture libraries," IEEE Transactions on Pattern Analysis and Machine Intelligence 23(9), pp. 947-963, 2001.

3. K. Koffka, Principles of Gestalt Psychology, Harcourt, New-York, 1935.

4. S. Sarkar and K. Boyer, "Perceptual organization in computer vision: a review and a proposal for a classificatory structure," IEEE Transactions on Systems, Man, and Cybernetics 23(2), pp. 388-399, 1993.

5. D. Lowe, Perceptual Organization and Visual Recognition, Kluwer, Boston, 1985.

6. A. Desolneux and J. Morel, "A grouping principle and four applications," IEEE Transactions on Pattern Analysis and Machine Intelligence 25(4), pp. 508-513, 2003.

7. S. Sarkar and K. Boyer, "Integration, inference, and management of spatial information using bayesian networks: Perceptual organization," IEEE Transactions on Pattern Analysis and Machine Intelligence 15(3), pp. 256-274, 1993.

8. S. Sarkar and K. Boyer, "A computational structure for preattentive perceptual organization : graphical enumeration and voting method," IEEE Transactions on Systems, Man, and Cybernetics 24(2), pp. 246267, 1994.

9. A. Ackermann, A. Massmann, S. Posh, G. Sagerer, and D. Schlüter, "Perceptual grouping of contour segments using markov random fields," Pattern Recognition and Image Analysis 7(1), pp. 11-17, 1997.

10. H. Kang and E. Walker, "Multilevel grouping: combining bottom-up and top-down reasoning for object recognition," in proc. of International Conference on Pattern Recognition (ICPR'94), pp. 559-562, 1994.

11. R. Mohan and R. Nevatia, "Perceptual organization for scene segmentation and description," IEEE Transactions on Pattern Analysis and Machine Intelligence 14(6), pp. 616-635, 1992.

12. V. Murino, C. Regazzoni, and G. Foresti, "Grouping as a searching process for minimum-energy configurations of labelled random fields," Computer Vision and Image Understanding 64(1), pp. 157-174, 1996.

13. K. Idrissi, G. Lavoue, J. Ricard, and A. Baskurt, "Object of interest-based visual navigation, retrieval, and semantic content identification system," Computer Vision and Image Understanding 94(1-3), pp. 271-294, 2004.

14. J. Luo and C. Guo, "Perceptual grouping of segmented regions in color images," Pattern Recognition 36(12), pp. 2781-2792, 2003.

15. G. Shafer, A Mathematical Theory of Evidence, Princeton University Press, 1976.

16. P. Vasseur, C. Pegard, E. Mouaddib, and L. Delahoche, "Perceptual organization approach based on dempster-shafer theory," Pattern Recognition 32(8), pp. 1449-1462, 1999.

17. T. Pavlidis and S. Horowitz, "Segmentation of plane curves," IEEE Transaction on Computers 23(8), pp. 860-870, 1974.

18. D. Comaniciu and P. Meer, "Robust analysis of feature spaces: color image segmentation," in proc. of Computer Vision and Pattern Recognition (CVPR'97), pp. 750-757, 1997. 